\title{
Online integrated solution to collect data, generate information and manage events in the human biomonitoring field
}

\author{
M. Fátima Reis ${ }^{\mathrm{a}, *}$, João Tedim ${ }^{\mathrm{b}}$, Pedro Aguiar ${ }^{\mathrm{c}}$, J. Pereira Miguel ${ }^{\mathrm{a}}$, \\ Ludwine Casteleyn $^{\mathrm{d}}$, Reinhard Joas ${ }^{\mathrm{e}}$, Birgit Van Tongelen ${ }^{\mathrm{f}}$ \\ ${ }^{a}$ Institute of Preventive Medicine, Lisbon Faculty of Medicine, Av. Prof. Egas Moniz, Edificio Egas Moniz, \\ Piso 0, Ala C, 1649-028 Lisbon, Portugal \\ ${ }^{\mathrm{b}}$ Senso Comum, Aveiro, Portugal \\ ${ }^{\mathrm{c}}$ National School of Public Health, New University of Lisbon, Portugal \\ ${ }^{\mathrm{d}}$ University of Leuven, Government of Flanders, Belgium \\ ${ }^{\mathrm{e}}$ BIPRO GmbH, ESBIO Coordination, Germany \\ ${ }^{\mathrm{f}} D G$ Environment, European Commission, Belgium
}

\begin{abstract}
In the ambit of Work Package 1 of the ESBIO Project, an online integrated solution to collect data, to generate information, and to manage mainly information-sharing events related with human biomonitoring within Europe has been designed and is being implemented. The present paper summarises the methodological approaches used by the authors as proposers, general promoters and disseminators of this strategic concept, as well as the first outcomes and future actions to be taken, in the short and longer term, to face present and future challenges to make this innovative solution happen.
\end{abstract}

(C) 2007 Elsevier GmbH. All rights reserved.

Keywords: Human biomonitoring; HBM; Online inventory; Overview; Web platform; ESBIO

\section{Introduction}

The overall objective of the Expert team to Support BIOmonitoring (ESBIO) Project (http:// www.eu-humanbiomonitoring.org) is the development of a coordinated approach to human biomonitoring (HBM) in Europe, in line with Action 3 of the EU Environment and Health $(\mathrm{E} \& \mathrm{H})$ Action Plan (European Commission, 2004) by providing strong scientific support for the pilot project to be launched at the end of 2006.

\footnotetext{
*Corresponding author. Tel.: + 351217985 100; fax: + 351217999487

E-mail address: mfreis@fm.ul.pt (Reis M. Fátima).
}

Since the EU E\&H Action Plan is based on the current state of the art of EU HBM activities, the ESBIO proposal aims to overcome specific deficiencies in the present situation, in particular by generating significant added value with an updated and extended inventory of EU HBM activities and databases and a concept of a EU platform for exchange of information, expertise and experiences among individuals and institutions performing HBM activities (ESBIO, 2005).

The ESBIO Project is being developed on the basis of dedicated work packages (WP) and defined deliverables that systematically contribute to the enhancement of the current state of the art of EU HBM activities. The overall objective of ESBIO WP 1 is: to establish an updated European inventory of past (last 10 years) and 
ongoing HBM activities and databases within Europe; to develop a concept on how to continue to update the established inventory; to promote an updated overview of biomonitoring in Europe in order to provide effective inputs to the EU pilot project; and to ensure that all relevant activities and stakeholders can be and remain integrated through an EU web platform.

Being essential that all useful approaches and data already in existence are used in an efficient way and that appropriate continuity is facilitated, the starting point to update the inventory has been, as originally planned, the inventory (TWG Biomonitoring of Children, 2004a) developed by the Technical Working Group (TWG) on Biomonitoring of Children, which has been set up by the European Commission in the framework of the "Environment and Health Strategy" (COM(2003)338final). The basis for this former inventory was data on ongoing or past (last 10 years) environment and health biomonitoring activities related to children within Europe, collected through a questionnaire sent to institutions and researchers all over Europe.

The questionnaire, developed and agreed upon by the TWG Biomonitoring of Children, was composed of 42 open questions addressing: the population under study; biomarkers and additional data collected; analysis and quality control procedures; strategies for data protection; data availability; communication at the individual and group level; identified problems and shortcomings; positive aspects and suggestions helpful for similar activities. The former inventory was the result of the analysis performed on basic data from the 103 returned questionnaires. A preliminary overview (TWG Biomonitoring of Children, 2004b) has also been compiled from the material submitted.

In returned questionnaires, some problems have been identified: on the one hand, responses to the questions have been provided with different levels of detail; on the other hand, available information has not been provided due to (a) time and effort constraints and (b) duplication with other questionnaires. Preparation of the preliminary overview from the inventory database has not been an easy task, mainly due to different levels of detail on provided responses to the open questions. Moreover, the static character of the data collection and corresponding analysis only permits an overview relative to a defined "point" on a time scale that cannot easily be updated on a more or less regular basis.

To overcome identified problems concerning both the inventory and the overview, a new strategy has been designed and implemented, with some specific features and objectives: (1) a maximum number of closed questions in the questionnaire, to avoid different levels of detail in equivalent responses; (2) an online and easy to use form (almost all answers by checkbox), to facilitate respondent efforts and reduce time taken to fill in the questionnaire; (3) an exhaustive and dynamic form, accessible to those involved in HBM activities, and capable of producing automatically distinct overviews for different users' profiles, to avoid the need for additional questionnaires on unaddressed aspects of HBM or for other users' requests.

The present paper aims to summarise the methodological approaches, used by some ESBIO members and other authors in the ambit of WP1, to develop and implement a strategic concept of an online integrated solution in the HBM field, to accomplish the following specific objectives: (1) to update the inventory and to continue updating it over time, even after the end of the ESBIO Project; (2) to create online facilities to produce automatic updated overviews, aiming to facilitate dissemination of generated information; and (3) to create and implement a web platform for exchange of HBM information, expertise and experiences, in order to ensure that all relevant HBM activities and data can be integrated and all stakeholders can share implemented facilities, to enable more significant information sharing and better translation into policies. Another objective of the present paper is the description of the first outcomes and future actions to be taken to make this solution happen.

\section{Methods}

\section{Updated inventory and overviews}

To update the inventory and corresponding overviews, working steps have included, in a first phase, an analysis of content (Bardin, 2004) of all 103 returned questionnaires of the former inventory. In this "analysis phase", all responses to every question were compared to each other, in order to identify a maximum number of specific keywords related to the question under analysis.

In a second phase, a systematic organisation of identified keywords in a coherent set of questions (the "new form") has been performed (Hill and Hill, 2002), having in mind three complementary objectives: (1) maintenance, as far as possible, of the structure of the "old" questionnaire; (2) short, clear and closed questions on all issues addressed by the "old" questionnaire; (3) availability of as many alternatives for answers as possible (based on identified keywords and from literature search) and opportunity for new alternatives in an additional field; in order to give all users equal possibilities to answer.

A third phase was simultaneously devoted to the construction of the website (http://www.hbm-inventory. org) to make the "new form" available online, and of a mailing list, as complete as possible, to send out the website link and to invite institutions and researchers involved in HBM activities to fill in the form for every 
HBM activity in progress or completed in the last 10 years. Invited users were also asked to send that link to other potential responders.

Based on this new form, designated as "European Inventory on Human Biomonitoring Activities (EIHBA)", another form has also been prepared to complement the updated inventory on HBM activities within Europe. The work began with a literature search, an extensive review on papers published in the last 10 years, mainly on surveillance and research involving HBM within Europe. Identically to what has been done with returned questionnaires, the content of collected papers has also been analysed, in order to identify relevant keywords. In a similar way, identified keywords have also been included in a coherent structure - another new form named "database of published articles on European human biomonitoring activities (DPA/EHBA)", to be completed for every published paper selected during the search.

These two databases can continue to be updated, by the responders to the EIHBA form and by ESBIO members or other elements that complete the DPA/ EHBA form with last published papers. This is the basic concept to continue updating the inventory during ESBIO's lifetime and the starting point to extend updating beyond the end of ESBIO.

From these databases, compilation of online overviews on HBM activities within Europe will be a relatively easy task. As a limit, depending on factors such as access profile, depth of search and required statistical analysis, updated overviews can be made broadly available "one click away".

\section{Concept for a EU web platform}

Concerning an EU platform on HBM (EP-HBM), the basic concept is a web management system, where all relevant stakeholders will gradually be integrated, starting from now and lasting beyond the ESBIO Project, mainly to build up knowledge from disperse data and to facilitate systematic improvement of the relationship between those involved in HBM activities and in policy making. However, other stakeholders are welcome and will play an important role in shaping up the final objectives of the EP-HBM.

The system is able to support online management of events and data, based on implemented functionalities, which are responsive to further developments and have already been used by the "new forms" and website, to update the inventory and overviews, as well as by the online organisation of ESBIO Conference.

Therefore, developing and implementing a EU web platform on the HBM field will be an effective way of taking concrete measures:

- to set up dedicated websites to collect data, to generate information and to manage mainly informa- tion-sharing events, completing in this way communication actions taken by specific groups;

- to provide regular information and, at the same time, to set out clear rules for participation and openness to all relevant stakeholders;

- to raise awareness of particular needs and constraints of all players in the HBM field, essential for their effective participation.

Maintenance of this system has to be ensured not only by the general promoters but especially by all those who will benefit from the promising platform outcomes: timely information generated by analysis of own and all other users' data; advantages offered by a common forum for exchange of knowledge and experiences between teams and countries, including international institutions and the international level beyond European borders; high potential for a better co-operation among scientific, societal and political sides. This means that to make the platform happen all players have to deliver what is expected from their side.

Given the needed conditions, the next steps will include a number of actions such as: motivation and mobilisation of all relevant players for continuous participation; development of additional information dissemination and deployment strategies; definition of user access profiles, implementing a practice for openness and transparency, respecting data contributors' permission; development of platform use indicators, monitoring, for example, user access and information delivery, in order to shape the proposed solution for present and future challenges in the HBM field.

\section{Results and discussion}

\section{Available and expected outcomes: analysis and discussion}

To date, the objectives of creating two online forms and one website to update inventory have already been accomplished and the link to this site has been sent to a relatively long (and still being updated) list of electronic addresses all over Europe. Concerning the dissemination of generated information, the first version of an online and easy to use statistical tool to allow registered users to produce their own updated online HBM overviews is already available. Further developments related to statistical analysis of inputted data will be provided upon request according to access profile.

Although improvements to the concept can still be made, the EU platform on HBM for exchange of information, expertise and experiences among individuals and/or institutions performing HBM activities, is in place. In fact, the dynamic concept of this platform 
has already provided the optimal support to run all contents and relationships included in the first version of the updated inventory on HBM and, on the other hand, a European Conference to get together providers of HBM information and to identify expertise and institutions performing HBM activities has been prepared based on the platform concept.

The almost full online organisation of this conference is thus an emerging feature of EP-HBM.

Last but not least, the concept on how to implement continuation of the updated inventory and corresponding overviews is in progress, representing another perspective of use of this EU web platform on HBM.

The first goal of this project was to build a data gathering tool that could reply to an urgent need to establish what work is being done in the HBM field and who is carrying it out.

This goal was accomplished through the development of a dynamic form platform that can generate the various components of a questionnaire available on the HBM-Inventory website.

Having determined what is being done and who is doing it, the second step was to build an online data analysis tool that can provide the platform users with more complex answers related to all the information (and not only the user information) inserted into the system.

The developed platform can now make available to all its users both global and specific responses to questions related to the European HBM activities landscape.

From analysis of implemented functionalities and expected further developments, two main conclusions can be drawn:

- in general, outcomes from the work done within ESBIO WP 1 have high potential to impact on the work of all following WPs;

- they are also able to provide the basis for all follow up work related to HBM activities (even after the end of the ESBIO Project), in particular in three different but complementary ways:

(1) continuous and online updating of the established inventory through efficient networking that significantly and recurrently enhances current HBM state of the art;

(2) regular dissemination of generated information (overviews according to predefined access profile) within the scientific community and to other relevant stakeholders involved in HBM research, surveillance or in policy making;

(3) establishment of a web platform that will bring together available knowledge, bridge the gap between scientific and political sides, and actively promote exchange of HBM experience and information among teams and countries, susceptible to extension at an international level beyond European borders and with international institutions.

Further steps have to be taken so that the full potential of the platform can be exploited by the biomonitoring community, but these will depend on the direction of the progress required and can be easily taken as the foundations have been solidly built in the form of the web platform.

\section{References}

Bardin, L., 2004. Análise de conteúdo, third ed. Edições 70, Lisbon.

ESBIO, 2005. Development of a coherent approach to human biomonitoring in Europe. Project Proposal. BIPRO GmbH, Munich.

European Commission, 2004. Communication from the Commission to the Council, the European Parliament, the European Economic and Social Committee "The European Environment and Health Action Plan 2004-2010", $\operatorname{COM}(2004) 416$ Final, Brussels.

Hill, M. and Hill, A., 2002. Investigação por questionário, second reviewed and corrected ed. Edições Sílabo Ltd., Lisbon.

TWG Biomonitoring of Children, 2004a. Baseline Report 'Biomonitoring of Children' in the Framework of the European Environment and Health Strategy, COM (2003) 338 Final <http://www.brussels-conference.org/ biomonitoring.htm $\rangle,\langle$ http://www.brusselsconference.org/ Download/baseline_report/BR_Biomonitoring_final.pdf $\rangle$

TWG Biomonitoring of Children, 2004b. Report on an Action Plan and Options for Action for 'Biomonitoring of Children' in the Framework of the European Environment and Health Strategy, $\operatorname{COM}(2003) 338$ Final $<$ http://europa.eu.int/comm/environment/health/finalreports_ en.htm $\rangle$. 